\title{
Radiation from a charged particle bunch in a circular waveguide having areas with corrugated and smooth walls
}

\author{
Aleksandra A. Grigoreva," Andrey V. Tyukhtin, and Viktor V. Vorobev \\ Saint Petersburg State University, 7/9 Universitetskaya embankment, Saint Petersburg, 199034, Russia \\ Sergey P. Antipov \\ Euclid Techlabs LLC, Bolingbrook, Illinois 60440, USA \\ and Argonne National Laboratory, Lemont, Illinois 60349, USA
}

(Received 14 March 2019; published 31 July 2019)

\begin{abstract}
We analyze the wave field of a charged particle bunch moving in a circular waveguide consisting of two parts: the first one has a corrugated wall and the other one has a smooth wall. It is assumed that the period and the depth of the corrugation are much less than the waveguide radius and wavelengths under consideration. The influence of corrugation on the electromagnetic field is analyzed using the method of equivalent boundary conditions (EBC). The cases of the bunch moving in both directions inside of such a waveguide are considered. We obtain analytical expressions for the main part of the field having discrete spectrum. Figures of a typical mode distribution of radiation are presented. We also perform numerical simulations in software package CST Particle Studio. Comparison of analytical and numerical results confirms the validity of the EBC method.
\end{abstract}

DOI: 10.1103/PhysRevAccelBeams.22.071303

\section{INTRODUCTION}

Periodic waveguide structures have achieved widespread use in many areas of physics and technology. Currently, essential attention has been paid to the use of corrugated waveguides as sources of terahertz radiation [1-5]. The main attention has been focused on the study of SmithPurcell radiation when the wavelengths are comparable to the structure period (e.g., [6]). However, it is also interesting to study long wave radiation with wavelengths which are much greater than the structure period $[1,4,5,7,8]$. The generation of radiation in the terahertz frequency range via ultrarelativistic electron bunch moving through a cylindrical periodic structure with small corrugations is described in $[1,4]$. The charge moving with arbitrary velocity is considered in the paper [7]. Note, as well, that methods of powerful nanosecond pulses generation in the millimeter wavelength range using oversized corrugated waveguides are currently studied $[9,10]$. The other field for application of periodic structures is connected with new nondestructive methods of bunches diagnostic. The bunch energy measuring by means of circular waveguide having walls with small rectangular cells is described in the paper [11].

*aleksandra.a.grigoreva@gmail.com

Published by the American Physical Society under the terms of the Creative Commons Attribution 4.0 International license. Further distribution of this work must maintain attribution to the author(s) and the published article's title, journal citation, and DOI.
This paper is devoted to the investigation of radiation produced by charged particle bunch intersecting the boundary between the corrugated waveguide area (corrugations have rectangular forms) and the area with smooth wall. Although problems with small rectangular corrugated waveguides have been considered earlier (e.g., [1,7,12]), the presence of a boundary between different waveguide areas was not taken into account.

The work has the following structure. The first part is devoted to formulation of eigenmodes of the periodical corrugated circular waveguide with respect to the condition that corrugation period and depth are small in comparison with waveguide radius and wavelength. These restrictions allow using the equivalent boundary conditions (EBC) [13] instead of setting the boundary conditions on the complicated corrugated surface. Further the analytical investigation of the wave field of the bunch is performed. The work is focused on the analysis of the so called free field which describes the influence of the boundary between corrugated and smooth areas. We also perform the direct numerical simulations in CST Particle Studio that allows testing the correctness of EBC usage.

It should be noted that there is another approach to finely corrugated structures which is equivalent to the method of EBC. It consists in replacing a corrugated structure with the conductive smooth surface covered with some thin material layer $[14,15]$. This idea was developed and corresponding surface impedance was calculated in work [12] where the corrugated waveguide is replaced with some smooth 
waveguide with a dielectric-magnetic layer. In order to demonstrate the two approaches equivalence, one can obtain, for example, the frequency of the mode excited by the relativistic bunch moving in the infinite corrugated waveguide in two ways. The calculation shows that for the structure considered in [12] (waveguide radius is equal to $3 \mathrm{~mm}$, corrugation period- $1 \mathrm{~mm}$, corrugation depth$0.45 \mathrm{~mm}$, corrugation width $-0.75 \mathrm{~mm}$ ) the frequency given in [12] differs from the frequency calculated with the use of EBC by $0.4 \%$ only. This small difference is easily explained by the rounded value of the equivalent dielectric constant given in [12]. Note that the results presented in the book [13] were obtained by Vainshtein and Sivov much earlier than all other analogues results in the area of finely corrugated surfaces electrodynamics.

\section{CORRUGATED WAVEGUIDE PROPER WAVES}

Before investigating the charge field, it is advisable to describe eigenmodes properties of a periodic perfectly conducting cylindrical waveguide with small corrugations (Fig. 1). We suppose that the following conditions are satisfied:

$$
d \ll a, \quad d_{3} \ll a, \quad d \ll \lambda, \quad d_{3} \ll \lambda,
$$

where $a$ means the waveguide radius, $d$ and $d_{3}$ are corrugation period and depth respectively, $\lambda$ is a mode wavelength. Note that the following analysis will be performed for the electromagnetic field Fourier transforms $\left(\vec{E}_{\omega}, \vec{H}_{\omega}\right)$. In addition, for further purpose we may confine ourselves with considering the transverse-magnetic (TM) eigenmodes only.

Inequalities (1) allow using $\mathrm{EBC}$ on the waveguide walls, i.e., impedance smooth surface [13], instead of consideration of strict boundary condition for corrugated surface. The EBC for TM-field has the form (hereinafter the cylindrical coordinate system is used)

$$
\left.E_{z \omega}\right|_{r=a}=\left.\eta^{m} H_{\varphi \omega}\right|_{r=a} .
$$

where $\eta^{m}$ is the impedance. In the geometry under consideration, it can be presented as [13]

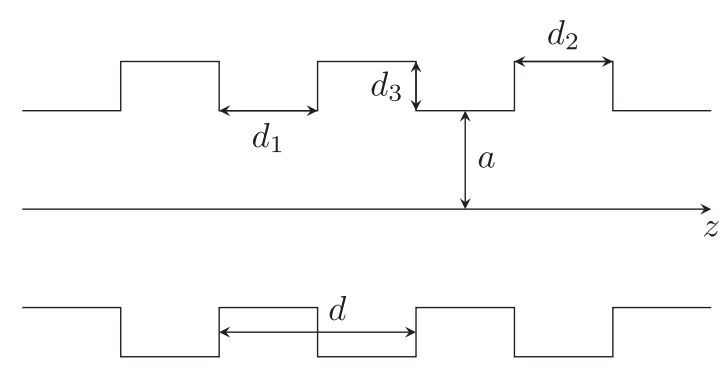

FIG. 1. Corrugated waveguide cross section.

$$
\eta^{m}=i \frac{\omega}{c}\left(\frac{d_{2} d_{3}}{d}-\delta \frac{c^{2} h^{2}}{\omega^{2}}\right)
$$

where $c$ is the speed of light in a vacuum, $h$ is a longitudinal wave number of the mode. The parameter $\delta$ is written in the form

$$
\begin{aligned}
\delta= & d_{3}+\frac{t d}{2 \pi} \int_{0}^{1 / \sigma} \frac{d u}{\sqrt{(1-u)(1-\sigma u)}(\sqrt{1-t u}+1)} \\
& +\frac{d}{2 \pi} \ln \left(\frac{\sigma-1}{\sigma}\right),
\end{aligned}
$$

where $t$ and $\sigma$ are determined from the following system of equations:

$$
\begin{aligned}
& \int_{0}^{t} \frac{\sqrt{t-u} d u}{\sqrt{u(1-u)(\sigma-u)}}=\pi \frac{d_{1}}{d}, \\
& \int_{t}^{1} \frac{\sqrt{u-t} d u}{\sqrt{u(1-u)(\sigma-u)}}=2 \pi \frac{d_{3}}{d} .
\end{aligned}
$$

The magnetic field Fourier transform must satisfy the equation

$$
\frac{\partial^{2} H_{\varphi \omega}}{\partial z^{2}}+\frac{1}{r} \frac{\partial}{\partial r}\left(r \frac{\partial H_{\varphi \omega}}{\partial r}\right)+\left(\frac{\omega^{2}}{c^{2}}-\frac{1}{r^{2}}\right) H_{\varphi \omega}=0
$$

and the conditions

$$
\begin{aligned}
\left|H_{\varphi \omega}\right|_{r=0} & <\infty, \\
\left.H_{\varphi \omega}\right|_{r=a} & =-\left.\left(\frac{i \eta^{m} \omega}{c}+\frac{1}{a}\right)^{-1} \frac{\partial H_{\varphi \omega}}{\partial r}\right|_{r=a} .
\end{aligned}
$$

The first condition means that the source is absent in the case of the problem for waveguide mode, and the second one follows from the EBC (2) and Maxwell equations. An arbitrary solution of the problem (6), (7) can be written as an expansion in series of eigenfunctions of transversal operator including differential operator

$$
L_{r}=\frac{1}{r} \frac{\partial}{\partial r}\left(r \frac{\partial}{\partial r}\right)-\frac{1}{r^{2}}+\frac{\omega^{2}}{c^{2}}
$$

and conditions (7). For each mode we have:

$$
H_{\varphi \omega n}=J_{1}\left(\chi_{n} r\right) \exp \left( \pm i h_{n}(\omega) z\right),
$$

where $h_{n}=\sqrt{\omega^{2} / c^{2}-\chi_{n}^{2}}$ is the mode longitudinal wave number. We define the physical sheet of the Riemann surface by condition $\operatorname{Im}\left(h_{n}\right) \geq 0$ for $\omega \in \mathbb{R}$, therefore the wave with the sign "+" propagates in the positive direction of the z-axis. The parameters $\chi_{n}$ are determined by the dispersion equation

$$
F_{\text {disp }} \equiv J_{1}(\chi a)-\frac{\chi c}{\omega\left|\eta^{m}\right|} J_{0}(\chi a)=0 .
$$


It should be noted that the functions $J_{1}(\chi r)$ possess the following orthogonality property (bar means the complex conjugation):

$$
\int_{0}^{a} \overline{J_{1}\left(\chi_{n} r\right)} J_{1}\left(\chi_{m} r\right) r d r=G_{m} \delta_{m n}
$$

where

$$
G_{m}=\frac{a^{2}}{2}\left[J_{1}^{2}\left(\chi_{m} a\right)+J_{0}^{2}\left(\chi_{m} a\right)\right]-\frac{a}{\chi_{m}} J_{0}\left(\chi_{m} a\right) J_{1}\left(\chi_{m} a\right)
$$

for $\chi_{m} \in \mathbb{R}$ and

$G_{m}=\frac{a^{2}}{2}\left[I_{1}^{2}\left(\left|\chi_{m}\right| a\right)-I_{0}^{2}\left(\left|\chi_{m}\right| a\right)\right]+\frac{a}{\left|\chi_{m}\right|} I_{0}\left(\left|\chi_{m}\right| a\right) I_{1}\left(\left|\chi_{m}\right| a\right)$

for $\chi_{m}=i\left|\chi_{m}\right|$. Ultimately, the electromagnetic field as a set of eigenmodes which can be written in the following form:

$$
\begin{gathered}
E_{z \omega}=\frac{i c}{\omega} \sum_{n=1}^{\infty} B_{n}(\omega) \chi_{n} J_{0}\left(\chi_{n} r\right) \exp \left( \pm i h_{n} z\right), \\
E_{r \omega}= \pm \frac{c}{\omega} \sum_{n=1}^{\infty} B_{n}(\omega) h_{n} J_{1}\left(\chi_{n} r\right) \exp \left( \pm i h_{n} z\right), \\
H_{\varphi \omega}=\sum_{n=1}^{\infty} B_{n}(\omega) J_{1}\left(\chi_{n} r\right) \exp \left( \pm i h_{n} z\right),
\end{gathered}
$$

where $B_{n}(\omega)$ are coefficients of the mode expansion.

\section{RADIATION OF A CHARGE MOVING FROM THE CORRUGATED AREA TO THE SMOOTH ONE}

Consider the radiation of a charge moving in a composite waveguide structure. The left semi-infinite area is a periodic corrugated waveguide, the other is a smooth waveguide (Fig. 2). The charge $q$ moves with uniform velocity $\vec{v}=c \beta \vec{e}_{z}$ along the waveguide axis from the corrugated area into the smooth one and intersects the transverse boundary $z=0$ at time $t=0$. The main goal is to investigate the radiation generated in the smooth waveguide area. The analytical treatment is based on the

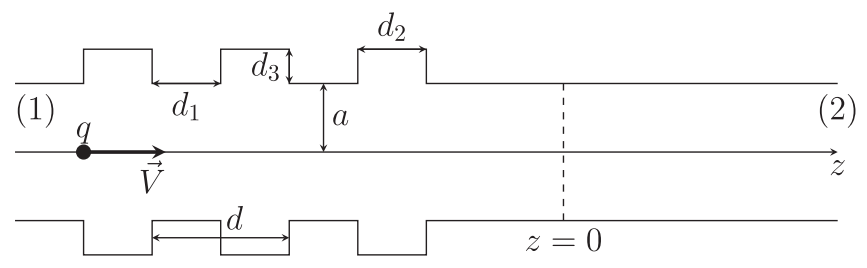

FIG. 2. Waveguide structure with corrugated (1) and smooth (2) areas. representation of the total electromagnetic field as a sum of so-called forced [with superscript $(q)$ ] and free [with superscript $(b)]$ fields in each waveguide area:

$$
\vec{E}_{1,2}=\vec{E}_{1,2}^{(q)}+\vec{E}_{1,2}^{(b)}, \quad \vec{H}_{1,2}=\vec{H}_{1,2}^{(q)}+\vec{H}_{1,2}^{(b)} .
$$

The forced field describes the electromagnetic field of the particle moving in an infinite, longitudinally homogeneous waveguide with properties of area (1) or (2). The free field, in turn, is excited because of the influence of the boundary between corrugated and smooth areas of the waveguide.

Forced fields for both areas have been investigated earlier $[7,16]$ :

$$
\begin{aligned}
E_{1,2 z \omega}^{(q)}= & -\frac{i q \omega}{\pi v^{2}}\left(1-\beta^{2}\right)\left(K_{0}(k r)+R_{1,2} I_{0}(k r)\right) \\
& \times \exp \left(\frac{i \omega z}{v}\right), \\
E_{1,2 r \omega}^{(q)}= & \frac{q k}{\pi v}\left[K_{1}(k r)-R_{1,2} I_{1}(k r)\right] \exp \left(\frac{i \omega z}{v}\right), \\
H_{1,2 \varphi \omega}^{(q)}= & \beta E_{1,2 r \omega}^{(q)} .
\end{aligned}
$$

Here subscripts 1 and 2 mean the corrugated waveguide and the smooth one respectively,

$$
\begin{aligned}
R_{1} & =-\frac{K_{0}(k a)+g k a K_{1}(k a)}{I_{0}(k a)-g k a I_{1}(k a)}, \quad R_{2}=-\frac{K_{0}(k a)}{I_{0}(k a)}, \\
k & =\frac{|\omega|}{v} \sqrt{1-\beta^{2}}, \quad g=\frac{1}{a\left(1-\beta^{2}\right)}\left(\beta^{2} \frac{d_{2} d_{3}}{d}-\delta\right),
\end{aligned}
$$

$I_{0,1}(x)$ are modified Bessel functions of the first kind, $K_{0,1}(x)$ are Macdonald functions.

The free field $\left\{\vec{E}^{(b)}, \vec{H}^{(b)}\right\}$ in areas (1) and (2) can be presented as an expansion by corrugated waveguide eigenmodes (11)-(2) and smooth waveguide eigenmodes correspondingly (such method is widely used in various problems [17-20]):

$$
\begin{aligned}
E_{1 z \omega}^{(b)} & =\frac{i c}{\omega} \sum_{n=1}^{\infty} B_{n}^{(1)}(\omega) \chi_{n} J_{0}\left(\chi_{n} r\right) \exp \left(-i h_{n}^{(1)} z\right), \\
E_{1 r \omega}^{(b)} & =-\frac{c}{\omega} \sum_{n=1}^{\infty} B_{n}^{(1)}(\omega) h_{n}^{(1)} J_{1}\left(\chi_{n} r\right) \exp \left(-i h_{n}^{(1)} z\right), \\
H_{1 \varphi \omega}^{(b)} & =\sum_{n=1}^{\infty} B_{n}^{(1)}(\omega) J_{1}\left(\chi_{n} r\right) \exp \left(-i h_{n}^{(1)} z\right), \\
E_{2 z \omega}^{(b)} & =\frac{i c}{\omega a} \sum_{n=1}^{\infty} B_{n}^{(2)}(\omega) \eta_{n} J_{0}\left(\eta_{n} \frac{r}{a}\right) \exp \left(i h_{n}^{(2)} z\right),
\end{aligned}
$$


$E_{2 r \omega}^{(b)}=\frac{c}{\omega} \sum_{n=1}^{\infty} B_{n}^{(2)}(\omega) h_{n}^{(2)} J_{1}\left(\eta_{n} \frac{r}{a}\right) \exp \left(i h_{n}^{(2)} z\right)$,

$H_{2 \varphi \omega}^{(b)}=\sum_{n=1}^{\infty} B_{n}^{(2)}(\omega) J_{1}\left(\eta_{n} \frac{r}{a}\right) \exp \left(i h_{n}^{(2)} z\right)$,

where $B_{n}^{(1,2)}(\omega)$ are unknown mode excitation coefficients, $\chi_{n}$ should be determined by dispersion equation (9), $\eta_{n}$ is the Bessel function zero $\left(J_{0}\left(\eta_{n}\right)=0\right), h_{n}^{(1)}=\sqrt{\omega^{2} / c^{2}-\chi_{n}^{2}}$ and $h_{n}^{(2)}=\sqrt{\omega^{2} / c^{2}-\eta_{n}^{2} / a^{2}}$ are longitudinal wavenumbers $\left[\operatorname{Im}\left(h_{n}^{(1,2)}\right) \geq 0\right.$ for $\left.\omega \in \mathbb{R}\right]$.

In order to find coefficients $B_{n}^{(1,2)}$ we use the continuity conditions for tangential components of the total electromagnetic field (12) at $z=0$. Further the obtained relations are multiplied by complex conjugate eigenfunction of corrugated waveguide $\overline{J_{1}\left(\chi_{m} r\right)}$ and integrated over the radial variable $r$ from 0 to $a$. After the series transformation using the orthogonal property (10) the following infinite system of equations can be formulated:

$$
\begin{gathered}
\sum_{n=1}^{\infty} M_{m n} B_{n}^{(2)}(\omega)=P_{m}(\omega), \quad m=1,2 \ldots, \\
M_{m n}=\frac{J_{1}\left(\eta_{n}\right)}{\eta_{n}^{2}-\bar{\chi}_{m}^{2} a^{2}}\left(h_{m}^{(1)}+h_{n}^{(2)}\right), \\
P_{m}(\omega)=\frac{q g \omega k}{\pi v c I_{0}(k a)}\left(1+h_{m}^{(1)} \frac{v}{\omega}\right) \\
\quad \times \frac{U_{m}}{a^{3} \bar{\chi}_{m} J_{0}\left(\bar{\chi}_{m} a\right)\left[I_{0}(k a)-g k a I_{1}(k a)\right]} .
\end{gathered}
$$

Here

$$
\begin{aligned}
U_{m}= & \int_{0}^{a} I_{1}(k r) J_{1}\left(\bar{\chi}_{m} r\right) r d r, \\
U_{m}= & \frac{i a^{2}}{2}\left[I_{0}^{2}(k a)-I_{1}^{2}(k a)-\frac{2}{k a} I_{0}(k a) I_{1}(k a)\right] \\
& \text { if } \chi_{m}=i k,
\end{aligned}
$$

and

$$
U_{m}=\frac{a k I_{0}(k a) J_{1}\left(\bar{\chi}_{m} a\right)-a \bar{\chi}_{m} I_{1}(k a) J_{0}\left(\bar{\chi}_{m} a\right)}{k^{2}+\bar{\chi}_{m}^{2}} \text { if } \chi_{m} \neq i k .
$$

Mode coefficients $B_{n}^{(1)}(\omega)$ for corrugated waveguide area are determined as follows

$$
\begin{aligned}
B_{m}^{(1)}(\omega)= & \frac{a^{3} \bar{\chi}_{m} J_{0}\left(\bar{\chi}_{m} a\right)}{G_{m}} \sum_{n=1}^{\infty} B_{n}^{(2)}(\omega) \frac{J_{1}\left(\eta_{n}\right)}{\eta_{n}^{2}-\bar{\chi}_{m}^{2} a^{2}} \\
& -\frac{q g k U_{m}}{\pi c G_{m} I_{0}(k a)\left[I_{0}(k a)-g k a I_{1}(k a)\right]},
\end{aligned}
$$

where parameters $G(\omega)$ are specified by Eq. (10).

Note that the forced field does not possess the wave part in the smooth vacuum waveguide area. The wave field in this area arises out of the free field only. Our analysis will be focused on the investigation of the discrete spectrum of the free field without consideration the continuous spectrum. The free field discrete part is determined by singularities of Fourier transforms (15a)-(15c), namely, by function poles. Analysis of expressions (16)-(18) reveals that each function $B_{n}^{(2)}(\omega)$ possesses the poles determined by the equation

$$
F_{w}(\omega) \equiv I_{0}(k a)-g k a I_{1}(k a)=0,
$$

This equation has been considered in the paper [7]. Note that real roots $\pm \omega_{1}$ of Eq. (21) determine the wave field frequency for infinite corrugated waveguide. Comparison of Eq. (21) with dispersion equation (9) at frequency $\omega_{1}$ reveals that $\chi_{1}\left(\omega_{1}\right)=i k\left(\omega_{1}\right)$.

In accordance with (15b) the free field radial component can be written as a sum of modes:

$E_{2 r}^{(b)}=\sum_{n=1}^{\infty} E_{2 r n}^{(b)}$

$E_{2 r n}^{(b)}=c J_{1}\left(\eta_{n} \frac{r}{a}\right) \int_{-\infty}^{\infty} \frac{B_{n}^{(2)}(\omega)}{\omega} h_{n}^{(2)}(\omega) \exp \left[\Phi_{n}(\omega)\right] d \omega$,

where $\Phi_{n}(\omega)=i h_{n}^{(2)} z-i \omega t$. In order to approximately calculate the free field with separation of the discrete spectrum, we use the steepest descent method (SDM) for integral in Eq. (22). The integral behavior investigation by SDM includes saddle points determination, steepest descent path (SDP) building and transformation of the initial integration path toward the SDP.

The equation for saddle points $\omega_{n}^{(s)}$ can be easily obtained in accordance with the equation

$$
\left.\frac{d \Phi_{n}(\omega)}{d \omega}\right|_{\omega=\omega_{n}^{(s)}}=0
$$

and it has the following form:

$$
z-v_{2 n}^{(g r)}(\omega) t=0
$$

where $v_{2 n}^{(g r)}(\omega)$ means the group velocity of the $n$th mode (22):

$$
v_{2 n}^{(g r)}(\omega)=c \sqrt{1-\frac{\eta_{n}^{2} c^{2}}{a^{2} \omega^{2}}}
$$




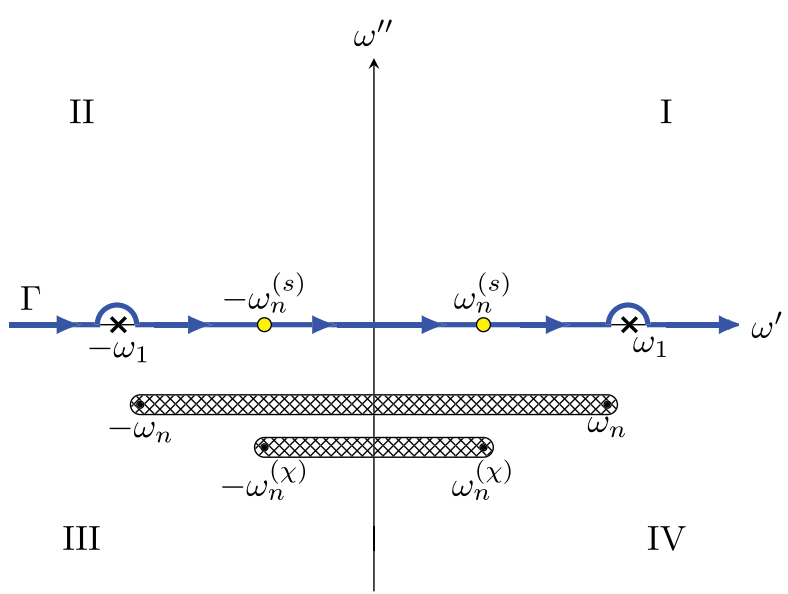

(a)

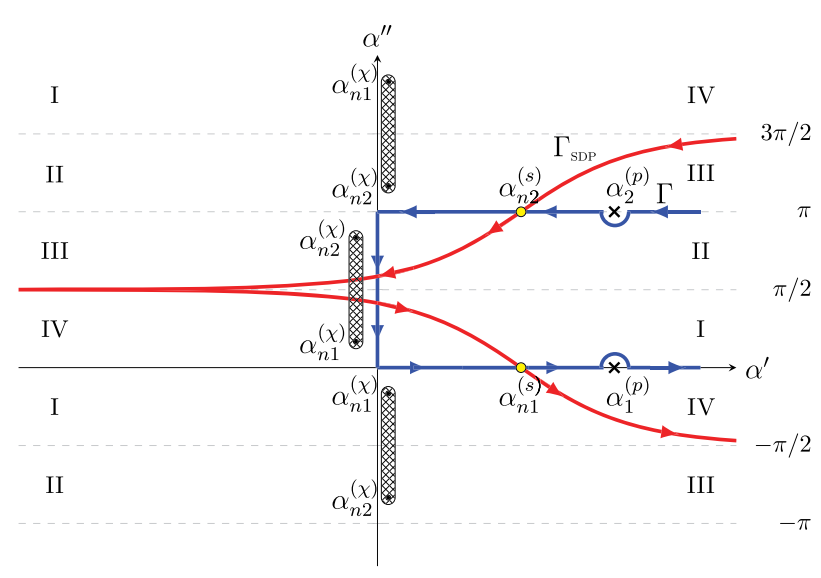

(b)

FIG. 3. The location of the integration path, the poles, the saddle points and the branch points on the complex plane $\omega$ (a) and $\alpha$ (b).

The integrand of each free field mode (22) possesses two saddle points

$$
\pm \omega_{n}^{(s)}= \pm \frac{\eta_{n} c^{2} t}{a \sqrt{c^{2} t^{2}-z^{2}}}
$$

Figure 3(a) shows the location of the initial integration path $\Gamma$, the saddle points $\pm \omega_{n}^{(s)}$, the poles $\pm \omega_{1}$, the branch points $\pm \omega_{n}^{(\chi)}$ determined by condition $h_{n}^{(1)}(\omega)=0$ and the branch points $\pm \omega_{n}= \pm \eta_{n} c / a$ determined by condition $h_{n}^{(2)}(\omega)=0$. The branch cuts are made along the lines $\operatorname{Re}\left(h_{n}^{(1)}\right)=0$ and $\operatorname{Re}\left(h_{n}^{(2)}\right)=0$ correspondingly. The change of variable $\omega=\omega_{n} \cosh (\alpha)$ results in the following: the branch cut between $-\omega_{n}$ and $\omega_{n}$ vanishes, the branch points $\pm \omega_{n}^{(\chi)}$ turn into $\alpha_{n 1,2}^{(\chi)}$, the saddle points turn into $\alpha_{n 1}^{(s)}=\operatorname{arcosh}\left(c t / \sqrt{c^{2} t^{2}-z^{2}}\right)$ and $\alpha_{n 2}^{(s)}=\alpha_{n 1}^{(s)}+i \pi$, the poles turn into $\alpha_{1,2}^{(p)}$. Also we obtain on the complex plane $\alpha$ the SDP $\Gamma_{\text {SPD }}$ [see Fig. 3(b)] consisting of two branch: through the saddle point $\alpha_{n 1}^{(s)}$

$$
\alpha^{\prime \prime}=-\operatorname{sgn}\left(\alpha^{\prime}-\alpha_{n 1}^{(s)}\right) \arccos \left[\frac{1}{\cosh \left(\alpha^{\prime}-\alpha_{n 1}^{(s)}\right)}\right]
$$

and through the saddle point $\alpha_{n 2}^{(s)}$

$$
\alpha^{\prime \prime}=\pi+\operatorname{sgn}\left(\alpha^{\prime}-\alpha_{n 1}^{(s)}\right) \arccos \left[\frac{1}{\cosh \left(\alpha^{\prime}-\alpha_{n 1}^{(s)}\right)}\right] .
$$

As one can see on Fig. 3, the poles $\pm \omega_{1}$ are crossed during transformation of the initial integration path toward the SDP under the condition $\left|\omega_{n}^{(s)}\right|<\left|\omega_{1}\right|$. Taking into account expressions (23) and (24) it can be shown that the poles crossing condition is the inequality

$$
z<v_{2 n}^{(g r)}\left(\left|\omega_{1}\right|\right) t
$$

Hence we obtain that the edge of the pole contribution existence moves in time with the group velocity of the "free" field mode.

The pole contributions to the integral (22) can be calculated using the residue theorem. After a series of mathematical transformations, we obtain the following expressions for components of the free field discrete part:

$$
\begin{aligned}
E_{2 z}^{(b w)}= & \frac{4 \pi c}{a \omega_{1}} \sum_{n=1}^{N_{2}} \Theta\left[v_{2 n}^{(g r)}\left(\omega_{1}\right) t-z\right] \eta_{n} J_{0}\left(\eta_{n} \frac{r}{a}\right) \\
& \times \operatorname{Re}\left\{\operatorname{Res}_{\omega=\omega_{1}}\left[B_{n}^{(2)}(\omega)\right] \exp \left[-i \omega_{1} t+i h_{n}^{(2)}\left(\omega_{1}\right) z\right]\right\},
\end{aligned}
$$

$$
\begin{aligned}
E_{2 r}^{(b w)}= & \frac{4 \pi c}{\omega_{1}} \sum_{n=1}^{N_{2}} \Theta\left[v_{2 n}^{(g r)}\left(\omega_{1}\right) t-z\right] h_{n}^{(2)}\left(\omega_{1}\right) J_{1}\left(\eta_{n} \frac{r}{a}\right) \\
& \times \operatorname{Im}\left\{\operatorname{Res}_{\omega=\omega_{1}}\left[B_{n}^{(2)}(\omega)\right] \exp \left[-i \omega_{1} t+i h_{n}^{(2)}\left(\omega_{1}\right) z\right]\right\},
\end{aligned}
$$

$$
\begin{aligned}
H_{2 \varphi}^{(b w)}= & 4 \pi \sum_{n=1}^{N_{2}} \Theta\left[v_{2 n}^{(g r)}\left(\omega_{1}\right) t-z\right] J_{1}\left(\eta_{n} \frac{r}{a}\right) \\
& \times \operatorname{Im}\left\{\operatorname{Res}_{\omega=\omega_{1}}\left[B_{n}^{(2)}(\omega)\right] \exp \left[-i \omega_{1} t+i h_{n}^{(2)}\left(\omega_{1}\right) z\right]\right\} .
\end{aligned}
$$

Here function $\Theta\left(v_{2 n}^{(g r)}\left(\omega_{1}\right) t-z\right)$ is conditioned by inequality (26) and determines the existence domain of the free field discrete part, $\operatorname{Res}_{\omega=\omega_{1}}\left(B_{n}^{(2)}(\omega)\right)$ is the function $B_{n}^{(2)}(\omega)$ 
residue, $N_{2}$ is the number of propagation modes in the smooth area generated at the frequency $\omega_{1}$.

The same analysis can be performed for the free field in the corrugated area $z<0$. The continuity conditions in this case

$$
\begin{aligned}
\left.\left(H_{1 \varphi \omega}^{(q)}+H_{1 \varphi \omega}^{(b)}\right)\right|_{z=0} & =\left.\left(H_{2 \varphi \omega}^{(q)}+H_{2 \varphi \omega}^{(b)}\right)\right|_{z=0}, \\
\left.\left(E_{1 r \omega}^{(q)}+E_{1 r \omega}^{(b)}\right)\right|_{z=0} & =\left.\left(E_{2 r \omega}^{(q)}+E_{2 r \omega}^{(b)}\right)\right|_{z=0},
\end{aligned}
$$

are multiplied by a function $J_{1}\left(\eta_{m} r / a\right)$ and integrated over the radial variable $r$ from 0 to $a$ with the use of orthogonality property

$$
\int_{0}^{a} J_{1}\left(\eta_{m} \frac{r}{a}\right) J_{1}\left(\eta_{n} \frac{r}{a}\right) r d r=\frac{a^{2}}{2} J_{1}^{2}\left(\eta_{m}\right) \delta_{m n} .
$$

After some mathematical transformation we obtain the following system of equations for the mode coefficients in the corrugated area

$$
\begin{gathered}
\sum_{n=1}^{\infty} \tilde{M}_{m n} B_{n}^{(1)}(\omega)=\tilde{P}_{m}, \quad m=1,2 \ldots \\
\tilde{M}_{m n}=\frac{\chi_{n} J_{0}\left(\chi_{n} a\right)}{\eta_{m}^{2}-\chi_{n}^{2} a^{2}}\left(h_{n}^{(1)}+h_{m}^{(2)}\right), \\
\tilde{P}_{m}=\frac{q g \omega k^{2}}{\pi c v F_{w}(\omega)} \frac{1}{\eta_{m}^{2}+k^{2} a^{2}}\left(1-h_{m}^{(2)} \frac{v}{\omega}\right),
\end{gathered}
$$

where $F_{w}(\omega)$ is equal to (21).

We are interested in calculation of the free field discrete part which is determined by contributions of the functions $B_{n}^{(1)}(\omega)$ poles. These poles, as before, are located at frequencies $\pm \omega_{1}$. Poles contributions can be obtained a similar to the analysis of the integral (22) way. Thus, we give only the final expressions for the free field discrete part in the corrugated area

$$
\begin{aligned}
E_{1 z}^{(b w)}= & \frac{4 \pi c}{\omega_{1}} \sum_{n=1}^{N_{1}} \Theta\left[v_{1 n}^{(g r)}\left(\omega_{1}\right) t-|z|\right] \\
& \times \operatorname{Re}\left\{\chi_{n}\left(\omega_{1}\right) J_{0}\left[\chi_{n}\left(\omega_{1}\right) r\right] \operatorname{Res}_{\omega=\omega_{1}}\left[B_{n}^{(1)}(\omega)\right]\right. \\
& \left.\times \exp \left[-i \omega_{1} t-i h_{n}^{(1)}\left(\omega_{1}\right) z\right]\right\}, \\
E_{1 r}^{(b w)}= & -\frac{4 \pi c}{\omega_{1}} \sum_{n=1}^{N_{1}} \Theta\left[v_{1 n}^{(g r)}\left(\omega_{1}\right) t-|z|\right] \operatorname{Im}\left\{h_{n}^{(1)}\left(\omega_{1}\right)\right. \\
& \times J_{1}\left[\chi_{n}\left(\omega_{1}\right) r\right] \operatorname{Res}_{\omega=\omega_{1}}\left[B_{n}^{(1)}(\omega)\right] \\
& \left.\times \exp \left[-i \omega_{1} t-i h_{n}^{(1)}\left(\omega_{1}\right) z\right]\right\},
\end{aligned}
$$

$$
\begin{aligned}
H_{1 \varphi}^{(b w)}= & 4 \pi \sum_{n=1}^{N_{1}} \Theta\left[v_{1 n}^{(g r)}\left(\omega_{1}\right) t-|z|\right] \operatorname{Im}\left\{J_{1}\left[\chi_{n}\left(\omega_{1}\right] r\right)\right. \\
& \left.\times \underset{\omega=\omega_{1}}{\operatorname{Res}}\left[B_{n}^{(1)}(\omega)\right] \exp \left[-i \omega_{1} t-i h_{n}^{(1)}\left(\omega_{1}\right) z\right]\right\},
\end{aligned}
$$

where function $\Theta\left(v_{1 n}^{(g r)}\left(\omega_{1}\right) t-|z|\right)$ is determined by the requirement of crossing the pole $\omega_{1}$ due to the transformation of the initial integration path toward the SDP, $v_{1 n}^{(g r)}(\omega)=\partial \omega / \partial h_{n}^{(1)}$ is the group velocity of the free field mode, $N_{1}$ is the number of propagation modes in the corrugated area.

Expressions (27a)-(27c), (31a)-(31c) allow calculating the averaged power $\left\langle\Pi_{1,2 z}\right\rangle$ of radiation (averaging is performed over time which is much greater than periods of all modes):

$$
\begin{aligned}
\left\langle\Pi_{1,2 z}\right\rangle & =\lim _{\tau \rightarrow \infty} \frac{1}{\tau} \int_{t}^{t+\tau} \Pi_{1,2 z} d t \\
\Pi_{1,2 z} & =\frac{c}{4 \pi} \int_{0}^{2 \pi} \int_{0}^{a} E_{1,2 r}^{(b w)} H_{1,2 \varphi}^{(b w)} r d r d \varphi .
\end{aligned}
$$

Here subscript 1 denotes the corrugated area and subscript 2 denotes the smooth one. Thus, we obtain the following expression for averaged power of radiation:

$$
\begin{aligned}
\left\langle\Pi_{1,2 z}\right\rangle & =\sum_{n=1}^{N_{1,2}}\left\langle\Pi_{1,2 z n}\right\rangle, \\
\left\langle\Pi_{1 z n}\right\rangle & =-\frac{4 \pi^{2} c^{2}}{\omega_{1}} h_{n}^{(1)}\left(\omega_{1}\right) G_{n}\left(\omega_{1}\right)\left|\operatorname{Res}_{\omega=\omega_{1}}\left[B_{n}^{(1)}(\omega)\right]\right|^{2}, \\
\left\langle\Pi_{2 z n}\right\rangle & =\frac{2 \pi^{2} c^{2} a^{2}}{\omega_{1}} J_{1}^{2}\left(\eta_{n}\right) h_{n}^{(2)}\left(\omega_{1}\right)\left|\operatorname{Res}_{\omega=\omega_{1}}\left[B_{n}^{(2)}(\omega)\right]\right|^{2},
\end{aligned}
$$

where $G_{n}(\omega)$ is specified by Eq. (10), $N_{1,2}$ means the number of propagating modes in the corrugated and smooth area correspondingly.

An example of the distribution of the averaged power $\left\langle\Pi_{1,2 z n}\right\rangle$ of the free field propagating modes is shown in Fig. 4 for different charge velocities. As one can see, in case the charge velocity is not very close to $c(\beta=0.85$ and $\beta=0.9$ ) several modes make a significant contribution to the averaged power in the smooth area. In the ultrarelativistic cases ( $\beta=0.95$ and $\beta=0.99999)$, however, the vast majority of the radiation power is transferred by the first mode only. Thus, it is possible to generate both singlemode and multi-mode radiation in the smooth area depending on the charge velocity. As for the averaged radiation power in the corrugated area, its value is less by two orders in comparison with the one in the smooth area. Thus, almost all the radiation generated in the corrugated structure penetrates into the smooth waveguide area. 

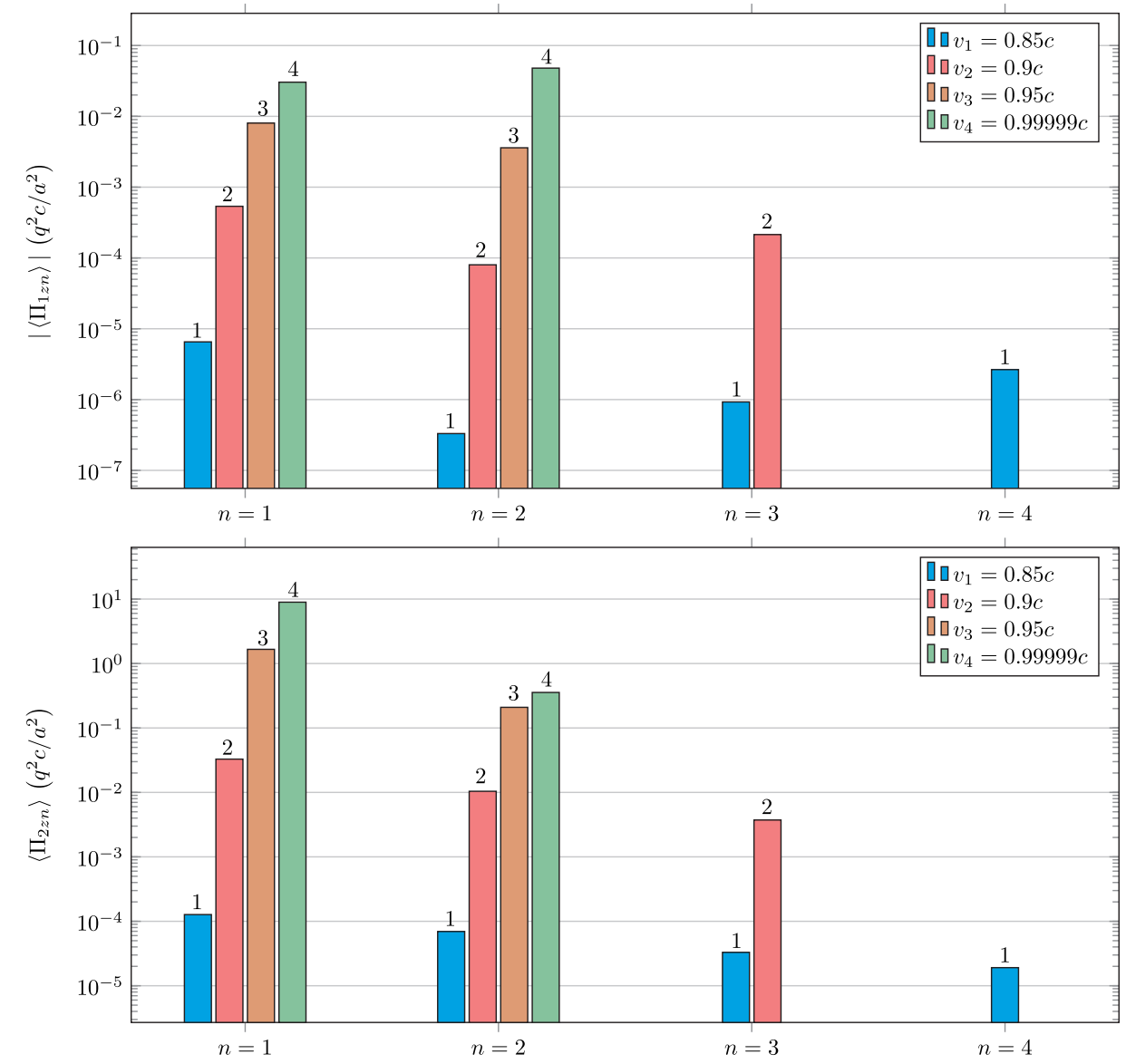

FIG. 4. Distribution of averaged power $\left|\left\langle\Pi_{1 z n}\right\rangle\right|$ (top) and $\left\langle\Pi_{2 z n}\right\rangle$ (bottom) of free field discrete part on a logarithmic scale for $a=1 \mathrm{~cm}, d_{1}=0.03 \mathrm{~cm}, d_{2}=0.04 \mathrm{~cm}, d_{3}=0.1 \mathrm{~cm}$. Different bars correspond to different charge velocities, $n$ means the number of propagating mode.

\section{RADIATION OF A CHARGE MOVING FROM THE SMOOTH AREA INTO THE CORRUGATED ONE}

Now we perform a similar analysis for the case of the reverse motion of the charge from the smooth waveguide area into the corrugated one. The application of the methods described above leads to the following system of equations:

$$
\sum_{n=1}^{\infty} A_{m n} B_{n}^{(2)}(\omega)=U_{m}, \quad m=1,2, \ldots
$$

where

$$
\begin{gathered}
A_{m n}=\frac{\chi_{n} J_{0}\left(\chi_{n} a\right)}{\eta_{m}^{2}-a^{2} \chi_{n}^{2}}\left(h_{m}^{(1)}+h_{n}^{(2)}\right), \\
U_{m}=-\frac{q \omega k^{2} g}{\pi c v F_{w}(\omega)}\left(1+h_{m}^{(1)} \frac{v}{\omega}\right) \frac{1}{\eta_{m}^{2}+k^{2} a^{2}},
\end{gathered}
$$

$B_{n}^{(2)}(\omega)$ is the free field mode expansion coefficient for the corrugated waveguide area, function $F_{w}(\omega)$ is determined by Eq. (21), $h_{m}^{(1)}=\sqrt{\omega^{2} / c^{2}-\eta_{m}^{2} / a^{2}}$ and $h_{n}^{(2)}=$ $\sqrt{\omega^{2} / c^{2}-\chi_{n}^{2}}\left(\operatorname{Im}\left(h^{(1,2)}\right) \geq 0\right.$ for $\left.\omega \in \mathbb{R}\right)$ are the free field longitudinal wave numbers (now indexes 1 and 2 denote to the smooth area and the corrugated one correspondingly). Coefficients for the free field in the smooth area can be calculated as follows

$$
\begin{aligned}
B_{m}^{(1)}(\omega)= & \frac{2 a}{J_{1}\left(\eta_{m}\right)}\left[\sum_{n=1}^{\infty} B_{n}^{(2)}(\omega) \frac{\chi_{n} J_{0}\left(\chi_{n} a\right)}{\eta_{m}^{2}-a^{2} \chi_{n}^{2}}\right. \\
& \left.+\frac{q g k^{2}}{\pi c F_{w}(\omega)\left(\eta_{m}^{2}+k^{2} a^{2}\right)}\right] .
\end{aligned}
$$

The main goal now is to analyze the wave field in the corrugated waveguide area. As follows from (12), it consists of two terms: the forced field [7] and the free field. Just as in the previous case, we will consider only the free field discrete part. 
Here we make a brief analysis of the free field discrete part by the example of radial component

$$
\begin{aligned}
E_{2 r}^{(b)}= & \sum_{n=1}^{\infty} E_{2 r n}^{(b)}, \\
E_{2 r n}^{(b)}= & c \int_{-\infty}^{+\infty} \frac{B_{n}^{(2)}(\omega)}{\omega} h_{n}^{(2)}(\omega) J_{1}\left[\chi_{n}(\omega) r\right] \\
& \times \exp \left[\Phi_{n}(\omega)\right] d \omega,
\end{aligned}
$$

where $\Phi_{n}(\omega)=i h_{n}^{(2)}(\omega) z-i \omega t$. The asymptotic behavior of the integral (39) can be considered by means of the $\mathrm{SDM}$, in much the same way as the integral (22). Since integrals (22) and (39) have a similar form, the equation for saddle points is identical to Eq. (23). Now, the group velocity of the free field eigenmode in the corrugated waveguide is written in the following form:

$$
v_{2 n}^{(g r)}(\omega)=\frac{\sqrt{\omega^{2} / c^{2}-\chi_{n}^{2}(\omega)}}{\omega / c^{2}-\chi_{n} \partial \chi_{n} / \partial \omega} .
$$

Free field discrete part is conditioned by singularities of the integrand (39) which are crossed at transformation of the initial integration path toward the SDP namely by poles of functions $B_{n}^{(2)}(\omega)$. Poles contributions, in turn, are determined by the residue theorem.

As one can see from (35)-(37) the coefficients $B_{n}^{(2)}(\omega)$ and right part of the system $\vec{U}$ has two pole type singularities $\pm \omega_{1}$ determined by the equation (21). For the purpose of further analysis convenience, we present the function $U_{m}(\omega)$ (37) as follows

$$
U_{m}(\omega)=\frac{P_{m}(\omega)}{F_{w}(\omega)},
$$

where

$$
P_{m}(\omega)=-\frac{q g \omega k^{2}}{\pi c v\left(\eta_{m}^{2}+k^{2} a^{2}\right)}\left(1+h_{m}^{(1)}(\omega) \frac{v}{\omega}\right),
$$

Functions $B_{n}^{(2)}(\omega)$ can be also presented in the form

$$
B_{n}^{(2)}(\omega)=\frac{\tilde{B}_{n}^{(2)}(\omega)}{F_{w}(\omega)}
$$

Thus, substitutions (41), (43) allow us to move from the system (35) to a more convenient system

$$
\sum_{n=1}^{\infty} A_{m n} \tilde{B}_{n}^{(2)}(\omega)=P_{m}(\omega), \quad m=1,2 \ldots
$$

It can be shown that the first column of the matrix $\hat{A}$ is proportional to the vector $\vec{P}$ at the frequency $\omega_{1}$

$$
A_{m 1}\left(\omega_{1}\right)=-\frac{i \pi c a}{q} I_{1}\left[k\left(\omega_{1}\right) a\right] P_{m}\left(\omega_{1}\right) .
$$

To derive the Eq. (45) we used the equality $\chi_{1}\left(\omega_{1}\right)=$ $i k\left(\omega_{1}\right)$ which results from coincidence between dispersion equation (9) and Eq. (21) at frequency $\omega_{1}$. Hence, the usage of the Cramers rule for solving the system (44) leads to the following result:

$\tilde{B}_{1}^{(2)}\left(\omega_{1}\right)=\frac{i q}{\pi c a I_{1}\left(k_{1} a\right)}, \quad \tilde{B}_{n}^{(2)}\left(\omega_{1}\right)=0 \quad(n \geq 2)$,

where $k_{1}=k\left(\omega_{1}\right)$. As follows from the expression (43), the non-nil $B_{n}^{(2)}(\omega)$ function residue at the pole $\omega_{1}$ is equal to

$$
\operatorname{Res}_{\omega=\omega_{1}}\left(B_{1}^{(2)}(\omega)\right)=\frac{i q}{\pi c a I_{1}\left(k_{1} a\right)}\left[\left.\frac{d F_{w}}{d \omega}\right|_{\omega_{1}}\right]^{-1} .
$$

The result is that the free field discrete part contains only one mode

$$
\begin{aligned}
E_{2 r}^{(b w)}= & -\frac{4 q}{a^{2}} \frac{I_{1}\left(k_{1} r\right)}{I_{1}^{2}\left(k_{1} a\right)} \frac{1}{\sqrt{1-\beta^{2}}} \frac{1}{1-g^{2} k_{1}^{2} a^{2}} \\
& \times \sin \left(\frac{\omega_{1} \zeta}{v}\right) \Theta(z) \Theta\left[v_{2,1}^{(g r)}\left(\omega_{1}\right) t-z\right] .
\end{aligned}
$$

Here $\zeta=z-v t, v_{2,1}^{(g r)}\left(\omega_{1}\right)$ means the group velocity of the first "free" field mode. Function $\Theta\left[v_{2,1}^{(g r)}\left(\omega_{1}\right) t-z\right]$ in expression (48) points out that the pole $\omega_{1}$ is crossed at transformation of the initial integration path toward the SDP under condition $z<v_{2,1}^{(g r)}\left(\omega_{1}\right) t$.

There is no difficulty to show that the free field discrete part $E_{2 r}^{(b w)}$ has the equal amplitude but the opposite sign compared to the forced field wave part [7] generated in the corrugated waveguide area behind the charge. So these fields compensate each other and the wave field under consideration is equal to zero in the area $z<v_{2,1}^{(g r)}\left(\omega_{1}\right) t$. Note that the same effect is observed in the case of vacuumdielectric junction in a waveguide [17] and in the case of vacuum-bilayer area junction [18]. 
In accordance with aforesaid the wave field in the corrugated waveguide area can be written in the following form:

$$
\begin{aligned}
E_{2 z}^{(w)}= & \frac{4 q}{a^{2}} \frac{I_{0}\left(k_{1} r\right)}{I_{1}^{2}\left(k_{1} a\right)} \frac{1}{1-g^{2} k_{1}^{2} a^{2}} \cos \left(\frac{\omega_{1} \zeta}{v}\right) \Theta(z) \Theta(-\zeta) \\
& \times \Theta\left[z-v_{2,1}^{(g r)}\left(\omega_{1}\right) t\right], \\
E_{2 r}^{(w)}= & \frac{4 q}{a^{2}} \frac{I_{1}\left(k_{1} r\right)}{I_{1}^{2}\left(k_{1} a\right)} \frac{1}{\sqrt{1-\beta^{2}}} \frac{1}{1-g^{2} k_{1}^{2} a^{2}} \sin \left(\frac{\omega_{1} \zeta}{v}\right) \Theta(z) \\
& \times \Theta(-\zeta) \Theta\left[z-v_{2,1}^{(g r)}\left(\omega_{1}\right) t\right], \\
H_{2 \varphi}^{(w)}= & \frac{4 q \beta}{a^{2}} \frac{I_{1}\left(k_{1} r\right)}{I_{1}^{2}\left(k_{1} a\right)} \frac{1}{\sqrt{1-\beta^{2}}} \frac{1}{1-g^{2} k_{1}^{2} a^{2}} \sin \left(\frac{\omega_{1} \zeta}{v}\right) \Theta(z) \\
& \times \Theta(-\zeta) \Theta\left[z-v_{2,1}^{(g r)}\left(\omega_{1}\right) t\right] .
\end{aligned}
$$

Here the product of the two theta-functions means that the wave field exists only in a bounded region $v_{2,1}^{(g r)}\left(\omega_{1}\right) t<$ $z<v t$ and its amplitude is equal to forced field amplitude.

\section{FIELD STRUCTURE}

Obtained results (27a)-(27c) and (49a)-(49c) can be generalized to the case of bunch possessing Gaussian longitudinal profile with volume charge density

$$
\rho^{(g)}=\frac{q}{2 \pi} \frac{\delta(r)}{r} \frac{\exp \left[-(z-v t)^{2} /\left(2 \sigma^{2}\right)\right]}{\sqrt{2 \pi} \sigma} .
$$

For this case, the field components of the discrete part are written in the form

$$
\begin{aligned}
\left\{E_{2 z}^{(g)(w)}, E_{2 r}^{(g)(w)}, H_{2 \varphi}^{(g)(w)}\right\}= & \left\{E_{2 z}^{(w)}, E_{2 r}^{(w)}, H_{2 \varphi}^{(w)}\right\} \\
& \times \exp \left(-\frac{\omega_{1}^{2} \sigma^{2}}{2 v^{2}}\right) .
\end{aligned}
$$

Figure 5 (top) shows the dependence of the wave field component $E_{2 r}^{(g)(w)}$ on z-coordinate calculated via analytical approach (51) at the observation moment $t=0.5 \mathrm{~ns}$ and at the point $r=0.5 \mathrm{~cm}$ in the case of bunch flying into the smooth waveguide area. The bunch is at the point $z_{q}=v t \approx 14.99 \mathrm{~cm}$ at the observation moment. The point $z=0 \mathrm{~cm}$ corresponds to the transverse boundary location. The free field discrete part contains two propagating modes at frequency $\nu_{1}=\omega_{1} /(2 \pi) \approx 31.5 \mathrm{GHz}$ at given parameters of the problem. As follows from the analytical investigation, the first free field mode exists in the area $z<z_{1} \quad\left(z_{1}=v_{2,1}^{(g r)}\left(\omega_{1}\right) t \approx 13.96 \mathrm{~cm}\right)$. The second mode end point is located at $z_{2}=v_{2,2}^{(g r)}\left(\omega_{1}\right) t \approx 8.24 \mathrm{~cm}$. The superposition of these two modes causes the beats in the area $z<z_{2}$. The case of the bunch flying into the
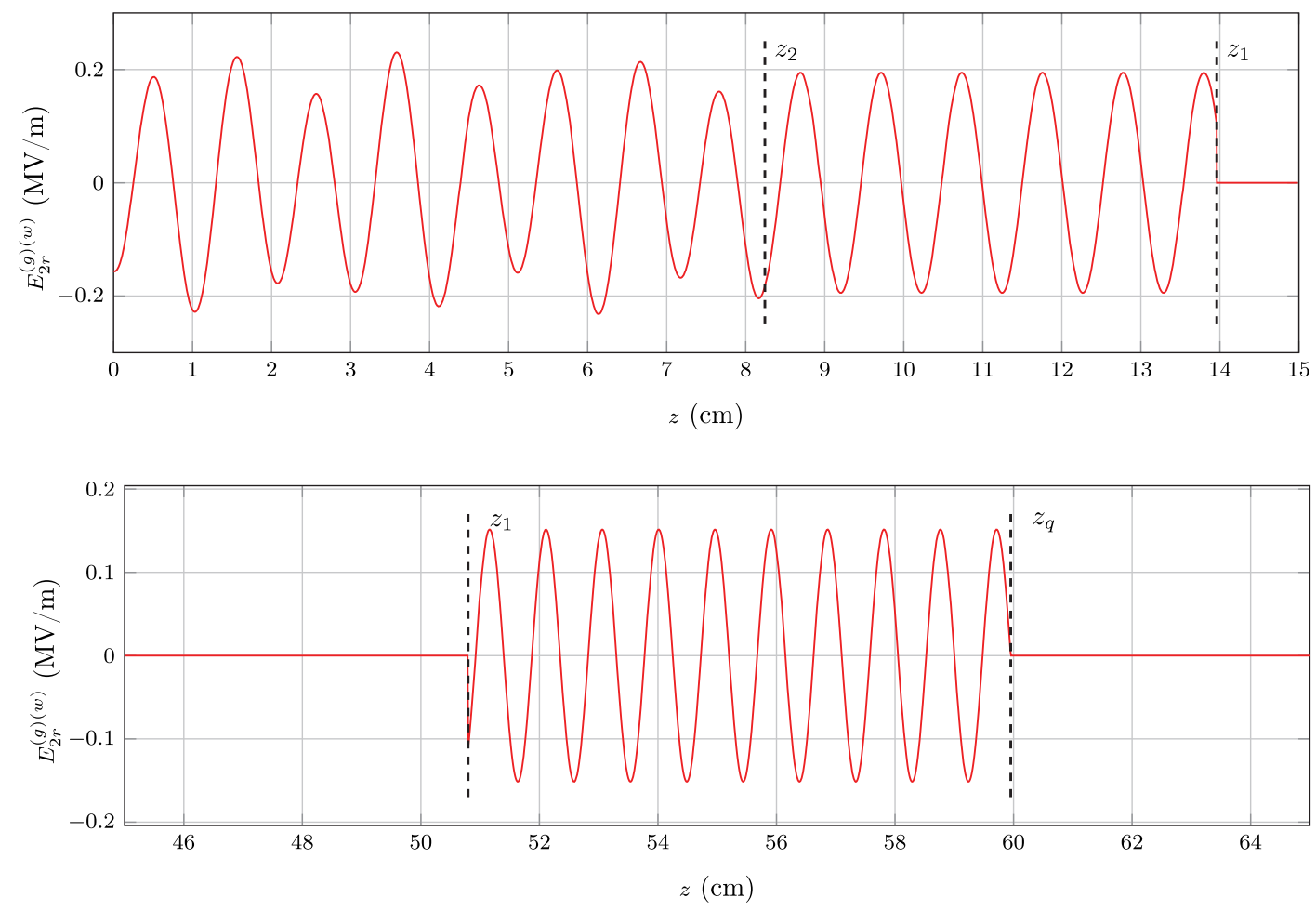

FIG. 5. The dependence of the wave field component $E_{2 r}^{(g)(w)}$ on the $z$-coordinate in the case of the bunch flying into the smooth area (top) and in the case of the bunch flying into the corrugated area (bottom). Waveguide and bunch parameters: $a=1 \mathrm{~cm}$, $d_{1}=d_{2}=0.05 \mathrm{~cm}, d_{3}=0.1 \mathrm{~cm}, q=1 \mathrm{nC}, \sigma=0.25 \mathrm{~cm}, v=0.9999 c, r=0.5 \mathrm{~cm}, t=0.5 \mathrm{~ns}$ (top) and $t=2 \mathrm{~ns}$ (bottom). 

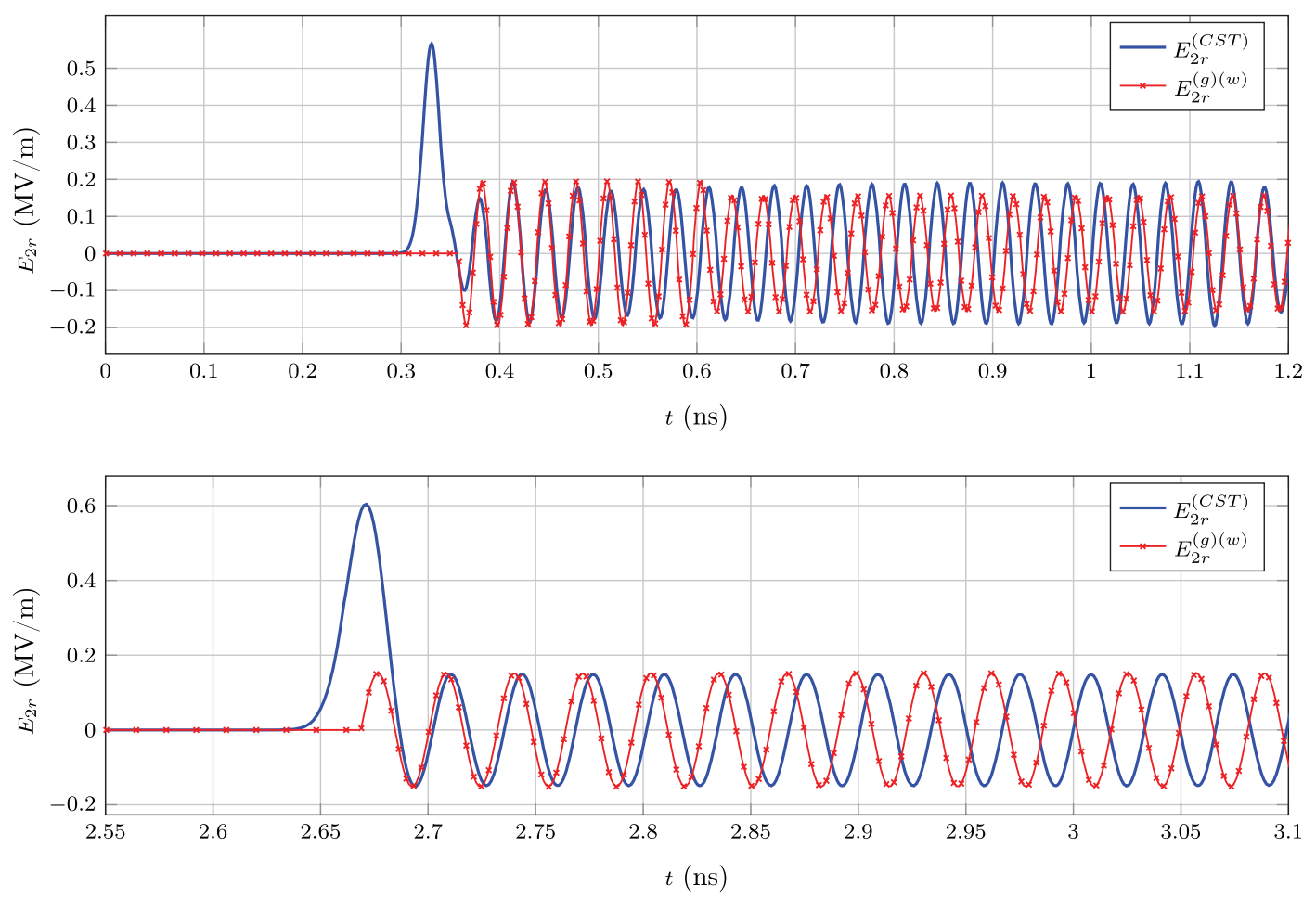

FIG. 6. Time dependence of the component $E_{2 r}^{(\mathrm{CST})}$ of the total electromagnetic field calculated via CST simulation (blue line) and the wave field $E_{2 r}^{(g)(w)}$ calculated via analytical approach (red marked line) in the case of the bunch flying into the smooth area (top) and in the case of the bunch flying into the corrugated area (bottom). Waveguide and bunch parameters are the same as in Fig. 5, observation point $r=0.5 \mathrm{~cm}, z=10 \mathrm{~cm}$ (top) and $z=80 \mathrm{~cm}$ (bottom).

corrugated waveguide area is presented in Fig. 5(bottom). The point $z_{q} \equiv v t$ denotes the bunch location at the observation moment $t=2 \mathrm{~ns}$. A distinctive feature of this case is that the wave field part with the discrete spectrum $E_{2 r}^{(g)(w)}$ exists in a restrictive area behind the bunch. According to [7], the forced field wave part exists at $z<z_{q}$. The free field discrete part according to (48) appears at point $z_{1} \equiv v_{2,1}^{(g r)}\left(\omega_{1}\right) t$. So it turns out that the wave field under consideration is identical to the forced field in the area $z_{1}<z<z_{q}$ and is equal to zero at $z<z_{1}$ because of compensation for the forced field wave part with a free field discrete part.

It should be noted that fields $E_{2 r}^{(g)(w)}$ presented in Fig. 5 have breakpoints at $z_{1,2}$ (top graph) and $z_{1, q}$ (bottom graph). Breakpoints are connected with the fact that analytical results (27a)-(27c) and (49a)-(49c) contain only the part of the wave field with the discrete spectrum. We used only the poles contributions in SDM for the free field discrete part. A comparison with numerical simulations however shows that the field part under consideration is the most significant (see Fig. 6).

Figure 6 shows the comparison between results calculated via analytical approach (51) and via numerical simulations in CST Particle Studio for the case of a Gaussian bunch. We used the waveguide model with the length of the smooth part $50 \mathrm{~cm}$ and the corrugated one is $100 \mathrm{~cm}$. The waveguide and bunch parameters are the same as in Fig. 5, the observation point is located at $r=0.5 \mathrm{~cm}$, $z=10 \mathrm{~cm}$ (top) and $z=80 \mathrm{~cm}$ (bottom). The open boundary conditions are set at the waveguide ends that ensures the absence of reflection from the ends. Note that EBC are not used in modeling. Instead, we consider the perfectly metallic waveguide with one part being corrugated. It should be noted that the first peak of the total field in Fig. 6 (for both cases) corresponds to the quasiCoulomb field of the bunch. Table I demonstrates frequency and amplitude of the radial field component of the total field $E_{2 r}^{(\mathrm{CST})}$ obtained using simulations and the wave field component $E_{2 r}^{(g)(w)}$ obtained analytically. The 1st and 2nd table rows refer to the cases of the bunch flying into the smooth area and into the corrugated one correspondingly. As can be seen, the discrepancy between analytical results and simulations does not exceed $4 \%$ for the frequencies and $6 \%$ for the amplitudes. These results confirm the validity of the EBC method, especially considering that the small parameters of the problem

$$
d / \lambda=d_{3} / \lambda \approx d / a=d_{3} / a=0.1
$$

are greater than obtained discrepancies. 
TABLE I. Key characteristics of the component $E_{2 r}^{(\mathrm{CST})}$ of the total electromagnetic field calculated via CST simulation and wave field $E_{2 r}^{(g)(w)}$ calculated via analytical approach (parameters are given in the capture if Fig. 6).

\begin{tabular}{lcclcc}
\hline \hline & \multicolumn{2}{c}{ Frequency, GHz } & & \multicolumn{2}{c}{ Amplitude, MV/m } \\
\cline { 2 - 3 } \cline { 5 - 6 } & Total field & Wave field & & Total field & Wave field \\
& $E_{2 r}^{(\mathrm{CST})}$ & $E_{2 r}^{(g)(w)}$ & & $E_{2 r}^{(\mathrm{CST})}$ & $E_{2 r}^{(g)(w)}$ \\
\hline a) & 30.2 & 31.5 & & 0.184 & 0.195 \\
b) & 30.2 & 31.5 & & 0.149 & 0.152 \\
\hline \hline
\end{tabular}

TABLE II. The key characteristics of the wave field component $E_{2 r}^{(g)(w)}$ in the case of corrugated waveguide area and in the case of bilayer waveguide area at point $r=0.5 \mathrm{~cm}, z=30 \mathrm{~cm}$. Bunch parameters are $q=1 \mathrm{nC}, v=0.9999 c, \sigma=0.25 \mathrm{~cm}$; corrugated area parameters are $a=1 \mathrm{~cm}, d_{1}=d_{2}=0.05 \mathrm{~cm}, d_{3}=0.1 \mathrm{~cm}$; bilayer area parameters are $a=1 \mathrm{~cm}, b=0.907 \mathrm{~cm}, \varepsilon_{d}=2$, $\varepsilon_{c}=1$

\begin{tabular}{lcc}
\hline \hline & Frequency, $\mathrm{GHz}$ & Amplitude, $\mathrm{MV} / \mathrm{m}$ \\
\hline Corrugated area & 31.5 & 0.152 \\
Bilayer area & 31.6 & 0.161 \\
\hline \hline
\end{tabular}

As shown in [14], the slightly corrugated waveguide can act like a smooth one covered with a thin dielectric layer. It would be interesting to make a similar comparison for our problem with the transverse boundary. In this connection, we compare the wave field generated by the Gaussian bunch flying from the smooth area to the corrugated one and the wave field generated by the bunch flying into the area having cylindrical dielectric layer and vacuum channel [18]. Table II shows wave field frequencies and amplitudes in these cases. Bunch parameters and the corrugated area characteristics are the same as in Fig. 5. For the case of waveguide having bilayer area, the permittivity of the dielectric layer and the channel radius are chosen so that the radiation frequency is close to the frequency of the wave field presented in Fig. 5. As can be seen from the Table II, the bunch generates almost equal wave fields in the corrugated area and in the bilayer one.

\section{CONCLUSION}

We have considered the electromagnetic field generation by the charged particle bunch passing through the two-part waveguide with the corrugated and smooth areas. The analytical solution has been obtained using EBC for corrugated wall. The work was mainly focused on the analysis of the discrete part of the field. It has been shown that frequencies of the forced field wave part and the free field discrete part are the same. Typical diagrams of the mode distribution of the averaged radiation power have been given.
In the case of the bunch flying from the corrugated waveguide area, it has been shown that averaged radiation power penetrating in the smooth area is much greater than the reflected radiation power. It has been also demonstrated that the decrease in bunch velocity leads to the more multifarious radiation structure. In the case of the bunch flying into the corrugated waveguide area we have shown that the discrete part of the wave field exists only in some bounded domain behind the bunch. The back front of the formed train moves with the group velocity of the mode. Numerical simulations of the problem performed using CST software package have confirmed the validity of the used method.

It was shown that the waveguide under consideration can behave in the same way as the smooth waveguide having the semi-infinite part with dielectric layer. However, in contrast with the smooth partially dielectric waveguide, the radiation in the partially corrugated waveguide is monochromatic (in the considered range of relatively low frequencies). Thus, one of the perspective applications of corrugated waveguides can be generation of monochromatic radiation.

\section{ACKNOWLEDGMENTS}

This work was supported by the Russian Science Foundation (Grant No. 18-72-10137).

[1] K. Bane and G. Stupakov, Terahertz radiation from a pipe with small corrugations, Nucl. Instrum. Methods Phys. Res., Sect. A 677, 67 (2012).

[2] A. Ponomarenko, K. Lekomtsev, A. Tishchenko, M. Strikhanov, and J. Urakawa, CST simulation of $\mathrm{THz}$ radiation from a channel with periodically variable radius, Nucl. Instrum. Methods Phys. Res., Sect. B 355, 160 (2015).

[3] A. Ponomarenko, A. Tishchenko, and M. Strikhanov, Thz polarization radiation from electrons passing corrugated dielectric tube under non-central propagation, Nucl. Instrum. Methods Phys. Res., Sect. B 402, 198 (2017), proceedings of the 7th International Conference Channeling 2016: Charged \& Neutral Particles Channeling Phenomena.

[4] K. Bane, G. Stupakov, S. Antipov, M. Fedurin, K. Kusche, C. Swinson, and D. Xiang, Measurements of terahertz radiation generated using a metallic, corrugated pipe, Nucl. Instrum. Methods Phys. Res., Sect. A 844, 121 (2017).

[5] M. Mineo and C. Paoloni, Corrugated rectangular waveguide tunable backward wave oscillator for terahertz applications, IEEE Trans. Electron Devices 57, 1481 (2010).

[6] P. Alexander, M. Ivanovich Ryazanov, M. Nikolaevich Strikhanov, and A. Tishchenko, Diffraction Radiation from Relativistic Particles (Springer, Berlin, Heidelberg, 2011). 
[7] A. Tyukhtin, V. Vorobev, E. Akhmatova, and S. Antipov, Radiation of charged particle bunches in corrugated waveguides with small period, J. Instrum. 13, C04009 (2018).

[8] A. Mostacci, F. Ruggiero, M. Angelici, M. Migliorati, L. Palumbo, and S. Ugoli, Wakefields due to surface waves in a beam pipe with a periodic rough surface, Phys. Rev. ST Accel. Beams 5, 044401 (2002).

[9] N. Ginzburg, V. Zaslavsky, I. Zheleznov, I. Zotova, A. Malkin, A. Sergeev, E. Kocharovskaya, and M. Yalandin, Mechanisms of cerenkov superradiance of extended electron bunches in oversized corrugated waveguides, Radiophys. Quantum Electron. 59, 461 (2016).

[10] N. S. Ginzburg, I. V. Zheleznov, A. M. Malkin, A. S. Sergeev, I. V. Zotova, and M. I. Yalandin, Terahertz superradiance of an extended electron bunch moving in an oversized corrugated cylindrical waveguide, in 2015 40th International Conference on Infrared, Millimeter, and Terahertz waves (IRMMW-THz), Hong Kong, China (IEEE, New York, 2015), pp. 1-1, https://www.doi.org/ 10.1109/IRMMW-THz.2015.7327464.

[11] A. Tyukhtin, Method of particle energy determination based on measurement of waveguide mode frequencies, J. Phys. Conf. Ser. 357, 012003 (2012).

[12] G. Stupakov and K. Bane, Surface impedance formalism for a metallic beam pipe with small corrugations, Phys. Rev. ST Accel. Beams 15, 124401 (2012).

[13] E. Nefedov and A. Sivov, Electrodynamics of Periodic Structures (Nauka, Moscow, 1977).
[14] A. Novokhatski and A. Mosnier, Wakefields of short bunches in the canal covered with thin dielectric layer, in Proceedings of the Particle Accelerator Conference, Vancouver, BC, Canada, 1997 (IEEE, New York, 1997), Vol. 2, pp. 1661-1663.

[15] K. L. F. Bane and A. Novokhatskii, The resonator impedance model of surface roughness applied to the LCLS parameters, Technical Report No. SLAC-AP-117, 1999.

[16] B. Bolotovskii, Theory of cherenkov radiation (III), Sov. Phys. Usp. 4, 781 (1962).

[17] T. Alekhina and A. Tyukhtin, Radiation of a charge in a waveguide with a boundary between two dielectrics, J. Phys. Conf. Ser. 357, 012010 (2012).

[18] A. Grigoreva, A. Tyukhtin, V. Vorobev, S. Galyamin, and S. Antipov, Radiation of a charge flying in a partially loaded dielectric section of a waveguide, Phys. Rev. Accel. Beams 21, 031302 (2018).

[19] T. Y. Alekhina and A. V. Tyukhtin, Reversed cherenkovtransition radiation in a waveguide partly filled with a left-handed medium, Radiat. Phys. Chem. 145, 43 (2018).

[20] A. Grigoreva, A. Tyukhtin, V. Vorobev, and S. Antipov, Radiation of a charge intersecting a boundary between a bilayer area and a homogeneous one in a circular waveguide, IEEE Trans. Microwave Theory Tech. 66, 49 (2018). 